\title{
P02-2-29 Poster session
}

\section{A search for effective drugs for treating chemotherapy-induced peripheral neuropathy}

\author{
Reiko Ishii-Nozawa, Yuya Umeda, Hidekazu Ikeya, Yoshihiko Haraguchi, Hajime Kagaya \\ Department of Clinical Pharmaceutics, Meiji Pharmaceutical University, Japan
}

Oxaliplatin (L-OHP) is a platinum-based anticancer drug used for treating colon cancer. Paclitaxel (PTX) is botanical in origin, and is used to treat several cancers such as ovarian, breast, and non-small cell lung cancers. Both of these drugs induce cold hypersensitivity and dysesthesia. Cold-sensitive transient receptor potential channels, TRPM8 and TRPA1, have been implicated as candidates mediating L-OHP- and PTX-induced cold allodynia and hyperalgesia. Fasudil, a cerebral ischemia-improving agent, is known to inhibit cerebral vasospasm by inhibiting Rho-kinase. Recently, the selective ROCK inhibitor fasudil demonstrated marked neuroprotective, neuroregenerative, and functional recovery effects in experimental animal models of stroke, spinal cord injury and demyelinating disease, thus providing a potential new therapeutic method for these conditions. In the present study, we examined the effects of fasudil on peripheral neuropathy in diabetic rats, and the inducing effects of L-OHP and PTX on cold allodynia in mice. Goto-Kakizaki (GK) rats were used as a diabetic model. Tail-flick test and acetone test, and von Frey tests for the thermally and mechanically induced pain models, respectively, were used to assess neuropathic pain in the diabetic rats. For chemotherapeutic drug experiments, 6-week-old ddY mice were used. L-OHP and PTX were injected intraperitoneally on day 1 only, whereas fasudil was administered intraperitoneally for five consecutive days. Cold allodynia was evaluated by the acetone test. These tests were performed on the date of administration and on five consecutive days after the administration of chemotherapeutic drugs. Fasudil, administered for 5 days to GK rats, improved the pain-related behavior. Single intraperitoneal administration of L-OHP and PTX induced cold allodynia on the third day in mice. Fasudil also suppressed the cold allodynia induced by the chemotherapeutic drugs. The Rho-kinase inhibitor fasudil can improve the peripheral nerve damage caused by diabetes peripheral neuropathy and chemotherapy-induced peripheral neuropathy. 